\title{
Anomalous triple-gauge-boson interactions in diboson production
}

\section{Mauro Chiesa*t, Ansgar Denner}

Julius-Maximilians-Universität Würzburg, Institut für Theoretische Physik und Astrophysik, D-97074 Würzburg, Germany.

E-mail: mauro.chiesa@physik.uni-wuerzburg.de,

ansgar.denner@physik.uni-wuerzburg.de

\section{Jean-Nicolas Lang}

Universität Zürich, Physik-Institut, CH-8057 Zürich, Switzerland.

E-mail: jlang@physik.uzh.ch

Diboson production is of prime interest at the LHC particularily due to its sensitivity to the gaugeboson self interaction, allowing to test its Standard Model prediction with high precision and to search for possible deviations with respect to the Standard Model. We report on the results of Ref. [1], where we computed WW, WZ and ZZ production (including the leptonic decays of the vector bosons) in the effective field theory framework at NLO QCD accuracy. The impact of the higher-dimensional operators is compared to the NLO QCD and NLO EW corrections in the Standard Model. Our calculation is the first application of RECOLA2 in the effective field theory framework.

Sixth Annual Conference on Large Hadron Collider Physics (LHCP2018)

4-9 June 2018

Bologna, Italy

* Speaker.

${ }^{\dagger}$ M. C. would like to thank the organizers for their kind invitation. 


\section{Introduction}

Searching for physics beyond the Standard Model (BSM) is one of the main tasks of the LHC. Since the new physics (NP) can manifest itself in mild deviations from the Standard Model (SM) background, high-precision measurements of SM processes represent a very important tool for the NP searches. Being sensitive to the gauge-boson self interaction, diboson production processes are used at the LHC to set limits on the anomalous (i.e. different from the SM) triple-gauge-boson interactions.

In order to point out small deviations from the SM expectation for diboson production, precise theoretical predictions for this process are mandatory. Two-loop QCD calculations as well as resummed predictions at NNLL+NNLO and NLO QCD+parton-shower results are available for diboson production. On the electroweak (EW) side, the one-loop EW corrections to all the fourlepton production processes have been computed. We refer to Ref. [1] for a detailed bibliography.

From an historical point of view, anomalous triple-gauge-boson interactions were first described in terms of anomalous couplings. In this approach, the most-general interaction term (compatible with a given set of symmetries) is added to the SM Lagrangian. From the resulting Lagrangian it is possible to derive the Feynman rules that can be employed in LO calculations. However, this approach does not provide any prescription to perform loop calculations.

The modern way to describe the anomalous triple-gauge-boson interaction is the SM effective field theory (SMEFT). In this approach the SM is considered as the low-energy limit of a UV-complete theory, whose Lagrangian $\mathscr{L}$ is in general unknown (different BSM models lead to a different ansatz for $\mathscr{L}$ ). The UV-complete theory in general includes new heavy degrees of freedom, however, they can be integrated out at low energies and $\mathscr{L}$ can be approximated by an effective Lagrangian as

$$
\mathscr{L}^{\text {eff. }}=\mathscr{L}^{\mathrm{SM}}+\sum_{i} \frac{c_{6}^{i}}{\Lambda^{2}} \mathscr{O}_{6}^{i}+\sum_{i} \frac{c_{8}^{i}}{\Lambda^{4}} \mathscr{O}_{8}^{i}+\cdots
$$

where $\mathscr{O}_{D}^{i}$ are the higher-dimensional operators describing the BSM interactions, $c_{D}^{i}$ are the corresponding Wilson coefficients and $\Lambda$ can be regarded as the NP scale.

As no significant deviation from the SM has been found so far at the LHC, the SMEFT provides a natural framework for the NP searches. Within the SMEFT it is possible to set limits on the NP effects without assuming a specific BSM model. Another interesting aspect of the SMEFT is the possibility to generalize this approach beyond the $\mathrm{LO}$ in the higher-dimensional operators.

In Ref. [1] we computed the processes pp $\rightarrow \mathrm{WW}\left(\rightarrow \mathrm{e}^{+} v_{\mathrm{e}} \mu^{-} \bar{v}_{\mu}\right), \mathrm{pp} \rightarrow \mathrm{WZ}\left(\rightarrow \mathrm{e}^{+} v_{\mathrm{e}} \mu^{+} \mu^{-}\right)$, and $\mathrm{pp} \rightarrow \mathrm{ZZ}\left(\rightarrow \mathrm{e}^{+} \mathrm{e}^{-} \mu^{+} \mu^{-}\right)$in the SMEFT framework at NLO QCD accuracy, but in the following we will focus on WZ production. We computed the NLO EW corrections to the processes under consideration as well as the contribution of the loop-induced gg $\rightarrow$ WW and gg $\rightarrow$ ZZ processes in order to compare these corrections with the impact of the anomalous triple-gauge-boson interactions. Our calculation relies on the code RECOLA2 [2, 3, 4] for the automated generation and the numerical evaluation of the tree-level and one-loop matrix elements. RECOLA2 uses the COLLIER [5] library for the tensor-integral reduction. We employed REPT1L [3] to derive the RECOLA2 model file describing the SM Lagrangian including the higher-dimensional 


\begin{tabular}{|l|l|l|l|}
\hline Setup & LO $[\mathrm{fb}]$ & NLO QCD $[\mathrm{fb}]$ & NLO EW [fb] \\
\hline $\mathrm{W}^{-} \mathrm{Z}$ ATLAS & $12.6455(9)_{-6.8 \%}^{+5.5 \%}$ & $23.780(4)_{-4.6 \%}^{+5.5 \%}$ & $11.891(4)_{-6.9 \%}^{+5.6 \%}$ \\
\hline $\mathrm{W}^{-} \mathrm{Z}$ CMS & $9.3251(8)_{-6.7 \%}^{+5.3 \%}$ & $17.215(4)_{-4.3 \%}^{+5.4 \%}$ & $8.870(2)_{-6.7 \%}^{+5.5 \%}$ \\
\hline $\mathrm{W}^{+} \mathrm{Z}$ ATLAS & $18.875(1)_{-6.4 \%}^{+5.2 \%}$ & $34.253(6)_{-4.3 \%}^{+5.3 \%}$ & $17.748(8)_{-6.5 \%}^{+5.3 \%}$ \\
\hline $\mathrm{W}^{+} \mathrm{Z}$ CMS & $14.307(1)_{-6.2 \%}^{+5.0 \%}$ & $26.357(6)_{-4.3 \%}^{+5.4 \%}$ & $13.600(4)_{-6.3 \%}^{+5.1 \%}$ \\
\hline
\end{tabular}

Table 1: Integrated cross section for WZ production at $\sqrt{s}=13 \mathrm{TeV}$ in the ATLAS and CMS setups. In the first column $\mathrm{W}^{+} \mathrm{Z}\left(\mathrm{W}^{-} \mathrm{Z}\right)$ is a short-hand notation for the process $\mathrm{pp} \rightarrow \mathrm{e}^{+} \nu_{\mathrm{e}} \mu^{+} \mu^{-}\left(\mathrm{pp} \rightarrow \mathrm{e}^{-} \bar{v}_{\mathrm{e}} \mu^{+} \mu^{-}\right)$. The numbers in parentheses correspond to the statistical error on the last digit.

operators relevant for diboson production starting from a UFO [6] model file produced by FEYNRULES [7, 8].

\section{Numerical results for $\mathrm{WZ}$ production}

Our results at the fiducial cross-section level for WZ production are collected in Tab. 1. We consider two event selections inspired by the ATLAS and CMS analyses of Refs. [9] and [10], respectively. The precise setups can be found in Ref. [1]. The NLO EW corrections are negative and moderate (of order $-6 \% /-5 \%$ ), while the NLO QCD corrections are enhanced by the opening of new channels with initial-state gluons and reach the value of $+80 \% /+90 \%$.

The differential distributions as a function of the transverse momentum of the $\mathrm{Z}$ boson $\left(p_{\mathrm{T}, \mu^{+} \mu^{-}}\right)$ and of the WZ transverse mass $\left(M_{\mathrm{T}}^{3 l v}\right)$ are shown in Fig. 1. The corrections follow the same pattern found at the cross-section level. The NLO EW corrections are enhanced at high $p_{\mathrm{T}}$ and/or $M_{\mathrm{T}}$ by
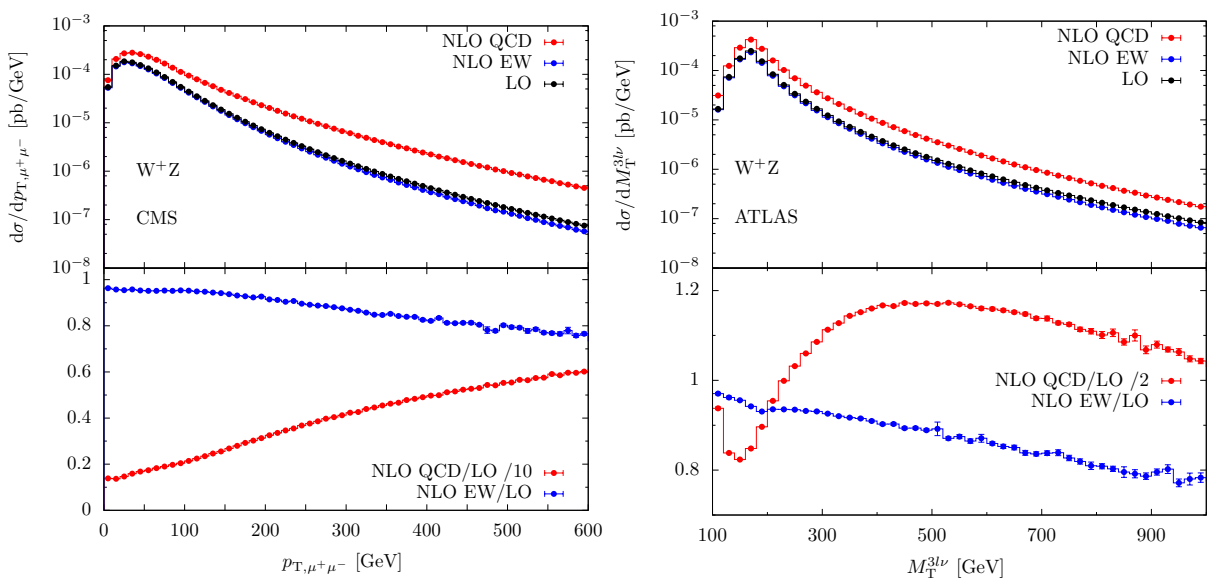

Figure 1: Differential distribution in the transverse momentum of the muon-antimuon pair $\left(p_{\mathrm{T}, \mu^{+} \mu^{-}}\right)$and in the WZ transverse mass $\left(M_{\mathrm{T}}^{3 l v}\right)$ for the process $\mathrm{pp} \rightarrow \mathrm{e}^{+} v_{\mathrm{e}} \mu^{+} \mu^{-}$at $\sqrt{s}=13 \mathrm{TeV}$. The NLO QCD predictions have been divided by a factor 10 (2) in the ratio NLOQCD/LO as a function of $p_{\mathrm{T}, \mu^{+} \mu^{-}}\left(M_{\mathrm{T}}^{3 l v}\right)$. 
the Sudakov logarithms. The NLO QCD corrections are positive and very large, in particular as a function of the $\mathrm{Z}$ boson $p_{\mathrm{T}}$.

The anomalous triple-gauge-boson interaction is parametrized in terms of dimension-six (Dim6) operators [11]:

$$
\begin{aligned}
\mathscr{O}_{W W W} & =-\frac{g_{\mathrm{w}}^{3}}{4} \varepsilon_{i j k} W_{\mu \nu}^{i} W^{v \rho j} W_{\rho}^{\mu k}, \\
\mathscr{O}_{W} & =-\mathrm{i} g_{\mathrm{w}}\left(D_{\mu} \Phi\right)^{\dagger} \frac{\tau_{k}}{2} W^{\mu \nu k}\left(D_{v} \Phi\right), \\
\mathscr{O}_{B} & =+\mathrm{i} \frac{g_{1}}{2}\left(D_{\mu} \Phi\right)^{\dagger} B^{\mu \nu}\left(D_{v} \Phi\right), \\
\mathscr{O}_{\widetilde{W} W W} & =+\frac{g_{\mathrm{w}}^{3}}{4} \varepsilon_{i j k} \widetilde{W}_{\mu \nu}^{i} W^{v \rho j} W_{\rho}^{\mu k}, \\
\mathscr{O}_{\widetilde{W}} & =+\mathrm{i} g_{\mathrm{w}}\left(D_{\mu} \Phi\right)^{\dagger} \frac{\tau_{k}}{2} \widetilde{W}^{\mu \nu k}\left(D_{v} \Phi\right),
\end{aligned}
$$

where $g_{\mathrm{w}}=e / s_{\mathrm{w}}, g_{1}=e / c_{\mathrm{w}}, \tau$ are the Pauli matrices and $\Phi$ stands for the Higgs doublet. For the corresponding Wilson coefficients we consider values

$$
\begin{array}{ll}
\frac{c_{W}^{+}}{\Lambda^{2}}=3 \times 10^{-6} \mathrm{GeV}^{-2}, & \frac{c_{B}^{+}}{\Lambda^{2}}=1.5 \times 10^{-5} \mathrm{GeV}^{-2}, \\
\frac{c_{W W W}^{+}}{\Lambda^{2}}=3 \times 10^{-6} \mathrm{GeV}^{-2}, & \frac{\tilde{c}_{W}^{+}}{\Lambda^{2}}=1 \times 10^{-6} \mathrm{GeV}^{-2}, \\
\frac{\tilde{c}_{W W W}^{2}}{\Lambda^{2}}=3 \times 10^{-6} \mathrm{GeV}^{-2} . &
\end{array}
$$

The values in Eq. (2.2) are in agreement with the current limits from the LHC.

According to Eq. (1.1), the cross sections and/or the differential distributions have the form

$$
\sigma=\sigma_{\mathrm{SM}^{2}}+\sigma_{\mathrm{SM} \times \mathrm{EFT} 6}+\sigma_{\mathrm{EFT}^{2}}+\sigma_{\mathrm{SM} \times \mathrm{EFT} 8}+\sigma_{\mathrm{EFT}^{2}}+\ldots,
$$

with

$$
\sigma_{\mathrm{SM} \times \mathrm{EFT} 6} \propto \frac{c_{6}}{\Lambda^{2}}, \quad \sigma_{\mathrm{EFT} 6^{2}} \propto \frac{c_{6}^{2}}{\Lambda^{4}}, \quad \sigma_{\mathrm{SM} \times \mathrm{EFT} 8} \propto \frac{c_{8}}{\Lambda^{4}}, \quad \sigma_{\mathrm{EFT} 8^{2}} \propto \frac{c_{8}^{2}}{\Lambda^{8}} .
$$

From Eqs. (2.3)-(2.4) it follows that in general it is not consistent to include the quadratic contributions in the Dim-6 operators if the Dim- 8 operators are neglected. On the other hand, there are models (such as the strongly interacting ones) where the $\sigma_{\mathrm{SM} \times \mathrm{EFT}}$ terms are subleading with respect to the $\sigma_{\mathrm{EFT6} 6^{2}}$ ones. For these reasons we show results for WZ production both with and without the contribution of the $\sigma_{\mathrm{EFT} 6^{2}}$ term.

Figure 2 shows the ratios

$$
\begin{aligned}
& R_{\text {lin }}^{\mathrm{LO}(\mathrm{NLO})}=\frac{\mathrm{d}\left(\sigma_{\mathrm{SM}^{2}}+\sigma_{\mathrm{SM} \times \mathrm{EFT}}\right)^{\mathrm{LO}(\mathrm{NLO}) \mathrm{QCD}} / \mathrm{d} X}{\mathrm{~d} \sigma_{\mathrm{SM}^{2}}^{\mathrm{LO}(\mathrm{NLO}) \mathrm{QCD}} / \mathrm{d} X}, \\
& R_{\text {quad }}^{\mathrm{LO}(\mathrm{NLO})}=\frac{\mathrm{d}\left(\sigma_{\mathrm{SM}^{2}}+\sigma_{\mathrm{SM} \times \mathrm{EFT}}+\sigma_{\mathrm{EFT}^{2}}\right)^{\mathrm{LO}(\mathrm{NLO}) \mathrm{QCD}} / \mathrm{d} X}{\mathrm{~d} \sigma_{\mathrm{SM}^{2}}^{\mathrm{LO}(\mathrm{NLO}) \mathrm{QCD}} / \mathrm{d} X},
\end{aligned}
$$

with $X=p_{\mathrm{T}, \mu^{+} \mu^{-}}$. Each line in the plots corresponds to a setup where only one of the Wilson coefficients is different from zero. The upper panels in the plots contain only the interference terms, while in the lower panels the quadratic contribution in the Dim-6 operators is included as 



Figure 2: Ratio $R_{\text {lin(quad) }}^{\mathrm{LO}(\mathrm{NLO})}$ as a function of the transverse momentum of the $\mathrm{Z}$ boson for the process $\mathrm{pp} \rightarrow$ $\mathrm{e}^{+} v_{\mathrm{e}} \mu^{+} \mu^{-}$. Each line corresponds to a setup where only one of the Wilson coefficients is different from zero. The black lines in the right plot correspond to the NLO EW corrections in the SM. In order to improve the plot readability, in the $R_{\text {lin }}^{\mathrm{LO}}$ ratio (upper panel, left plot) the curve labeled with $c_{W}^{+} / 4$ corresponds to our predictions where the $c_{W}^{+}$coefficient has been divided by a factor 4 .

well. The two main results are shown in Fig. 2: on the one hand, the largest contribution comes in general from the quadratic term and, on the other hand, the sensitivity to the Dim-6 operators decreases at NLO QCD. The reduction in the sensitivity can be traced back to the fact that the contribution of the Dim-6 operators increases with the centre-of-mass energy of the WZ system, while the real QCD radiation tends to reduce the centre-of-mass energy of the diboson system. As pointed out in Ref. [12] for on-shell vector bosons, the contribution of the $\mathscr{O}_{W W W}$ operator is suppressed at LO, but not at NLO QCD.

\section{Conclusions}

In Ref. [1] we computed WW, WZ, and ZZ production (including the leptonic decays of the vector bosons) in the SMEFT framework at NLO QCD accuracy. The anomalous $W W \gamma$ and $W W Z$ interaction was described in terms of Dim-6 operators, while Dim- 8 operators were used for the parametrization of the neutral triple-gauge-boson interactions. For all these processes we found that the sensitivity to the higher-dimensional operators is reduced at NLO QCD. We also found that the largest contributions come in general from the quadratic terms in the Dim-6 operators (Dim-8 operators for ZZ production).

\section{Acknowledgments}

The work of M.C. and A.D. was supported by the German Science Foundation (DFG) under reference number DE 623/5-1. J.-N. Lang acknowledges support from the Swiss National Science Foundation (SNF) under contract BSCGI0-157722. 


\section{References}

[1] M. Chiesa, A. Denner and J. N. Lang, Anomalous triple-gauge-boson interactions in vector-boson pair production with RECOLA2, Eur. Phys. J. C 78 (2018) no.6, 467 [arXiv:1804.01477 [hep-ph]].

[2] S. Actis, A. Denner, L. Hofer, J. N. Lang, A. Scharf and S. Uccirati, RECOLA: REcursive Computation of One-Loop Amplitudes, Comput. Phys. Commun. 214 (2017) 140 [arXiv:1605.01090 [hep-ph]].

[3] A. Denner, J. N. Lang and S. Uccirati, RECOLA2: REcursive Computation of One-Loop Amplitudes 2, Comput. Phys. Commun. 224 (2018) 346 [arXiv:1711.07388 [hep-ph]].

[4] A. Denner, J. N. Lang and S. Uccirati, NLO electroweak corrections in extended Higgs Sectors with RECOLA2, JHEP 1707 (2017) 087 [arXiv:1705.06053 [hep-ph]].

[5] A. Denner, S. Dittmaier and L. Hofer, COLLIER: a fortran-based Complex One-Loop LIbrary in Extended Regularizations, Comput. Phys. Commun. 212 (2017) 220 [arXiv:1604.06792 [hep-ph]].

[6] C. Degrande, C. Duhr, B. Fuks, D. Grellscheid, O. Mattelaer and T. Reiter, UFO - The Universal FeynRules Output, Comput. Phys. Commun. 183 (2012) 1201 [arXiv:1108.2040 [hep-ph]].

[7] N. D. Christensen and C. Duhr, FeynRules - Feynman rules made easy, Comput. Phys. Commun. 180 (2009) 1614 [arXiv:0806.4194 [hep-ph]].

[8] A. Alloul, N. D. Christensen, C. Degrande, C. Duhr and B. Fuks, FeynRules 2.0 - A complete toolbox for tree-level phenomenology, Comput. Phys. Commun. 185 (2014) 2250 [arXiv:1310.1921 [hep-ph]].

[9] G. Aad et al. [ATLAS Collaboration], Measurements of $W^{ \pm} Z$ production cross sections in $p p$ collisions at $\sqrt{s}=8 \mathrm{TeV}$ with the ATLAS detector and limits on anomalous gauge boson self-couplings, Phys. Rev. D 93, no. 9, 092004 (2016) [arXiv:1603.02151 [hep-ex]].

[10] V. Khachatryan et al. [CMS Collaboration], Measurement of the WZ production cross section in pp collisions at $\sqrt{s}=7$ and $8 \mathrm{TeV}$ and search for anomalous triple gauge couplings at $\sqrt{s}=8 \mathrm{TeV}$, Eur. Phys. J. C 77 (2017) no.4, 236 [arXiv:1609.05721 [hep-ex]].

[11] C. Degrande, N. Greiner, W. Kilian, O. Mattelaer, H. Mebane, T. Stelzer, S. Willenbrock and C. Zhang, Effective Field Theory: A Modern Approach to Anomalous Couplings, Annals Phys. 335 (2013) 21 [arXiv:1205.4231 [hep-ph]].

[12] A. Azatov, J. Elias-Miro, Y. Reyimuaji and E. Venturini, Novel measurements of anomalous triple gauge couplings for the LHC, JHEP 1710 (2017) 027 [arXiv:1707.08060 [hep-ph]]. 